\title{
EFFECT OF MAGNETIC PERTURBATIONS ON DIVERTOR SCRAPE-OFF WIDTH
}

BY

A. H, BOOZER AND A, B, RECHESTER

\section{PLASMA PHYSICS LABORATORY}

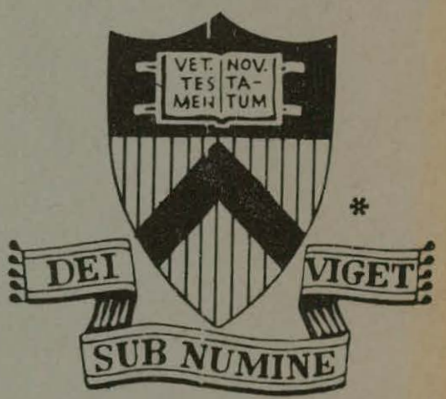

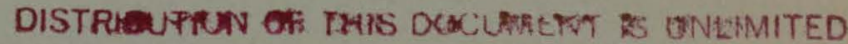

\section{PRINCETON \\ UNIVERSITY PRINCETON, NEW JERSEY}

This work was supported by U. S. Energy Research and Development Administration Contract E(11-1)-3073. Reproduction, translation, publication, use and disposal, in whole or in part, by or for the United States Government is permitted. 


\section{DISCLAIMER}

This report was prepared as an account of work sponsored by an agency of the United States Government. Neither the United States Government nor any agency Thereof, nor any of their employees, makes any warranty, express or implied, or assumes any legal liability or responsibility for the accuracy, completeness, or usefulness of any information, apparatus, product, or process disclosed, or represents that its use would not infringe privately owned rights. Reference herein to any specific commercial product, process, or service by trade name, trademark, manufacturer, or otherwise does not necessarily constitute or imply its endorsement, recommendation, or favoring by the United States Government or any agency thereof. The views and opinions of authors expressed herein do not necessarily state or reflect those of the United States Government or any agency thereof. 


\section{DISCLAIMER}

Portions of this document may be illegible in electronic image products. Images are produced from the best available original document. 
NOTICE

This report was prepared as an account of work sponsored by the United States Government. Neither the United States nor the United States Energy Research and Development Administration, nor any of their employees, nor any of their contractors, subcontractors, or their employees, makes any warranty, express or implied, or assumes any legal liability or responsibility for the accuracy, completeness or usefulness of any information, apparatus, product or process disclosed, or represents that its use would not infringe privately owned rights.

Printed in the United States of America.

Available from

National Technical Information Service

U. S. Department of Commerce 5285 Port Royal Road

Springfield, Virginia 22151

Price: Printed Copy $\$ \ldots$; Microfiche $\$ 3.00$

*Pages

$1-50$

51-150

$151-325$

326-500

501-1000
NTIS

Selling Price

$\$ 4.00$

5.45

7.60

10.60

13.60 


\title{
Effect of Magnetic Perturbations \\ On Divertor Scrape-off width
}

\author{
by \\ A. H. Boozer and A. B. Rechester
}

Plasma Physics Laboratory, Princeton University

Princeton, New Jersey 08540 


\title{
Effect of Magnetic Perturbations On Divertor Scrape-Off Width
}

\author{
A. H. Boozer and A. B. Rechester \\ Plasma Physics Laboratory, Princeton University \\ Princeton, New Jersey 08540
}

\begin{abstract}
A general method is developed for calculating the width, due to magnetic perturbations, of the scrape-off layer of a tokamak with a divertor. The general method is applied to a simple analytic model of a divertor to indicate the magnitude of the effects that would be expected.
\end{abstract}




\section{INTRODUCTION}

A poloidal divertor may bè needed in tokamak react,ors to maintain the low impurity levels required for fusion. The primary function of the divertor is to prevent plasma diffusing across the field lines from reaching the chamber walls. Plasma reaching the walls not only stirs up impurities, but also: adds to the neutral density in the region about the plasma. A: high neutral density can, through charge exchange, produce a flux of energetic particles on the walls creating additional impurities. A divertor leads plasma from the edge of the discharge through a small sio't in the chamber walls into a separate pumping chamber (Fig. 1). This arrangement not only prevents hot'plasma from reaching the walls; it also reduces the neutral density around the plasma. When a neutral hits the discharge, it has a good chance of being ionized before exiting. Once it is ionized it can be removed from the plasma chamber by the divertor. For the divertor to work effectively the scrapeoff layer (the region of open field lines, penetrated by the plasma), must be narrow: The width of the scrapeoff layer is quite different near the $x$. point than away from it. If $\delta$ is the fraction of the plasma radius occupied by the scrapeoff layer away from the $x$ point, then near the $x$ point that fraction is approximately $\delta^{1 / 2}$. Diffusion, whether classical or microturbulent, gives $\delta \sim 10^{-2}$. The classical diffusion scrapeoff width $\delta_{\mathrm{Cl}}$ is determined, by an 
ion only being able to move a drift orbit width as it flows down the field lines and out the divertor ${ }^{l}$ (the classical mean free path should be greater than the length of the field lines). Using the maximum ion drift orbit, we have $\delta_{\mathrm{Cl}} \simeq 3\left(q / \varepsilon^{1 / 2}\right) \mathrm{r}_{\mathrm{i}} / \mathrm{a}$ with $\varepsilon$ the inverse aspect ratio, $r_{i}$ the ion gyroradius, and a the minor radius. The safety factor q goes to infinity at the separatrix between open and closed lines. However, the singularity is quite weak being only logarithmic. In the model described in the paper, for distances of order $10^{-2}$ of the plasma radius away from the separatrix, $q \simeq q_{0}$ with $q_{0}$ the $q$ value at the physical location of the $x$ point with the divertor coil turned off. For reactor parameters $\mathrm{T}_{\mathrm{i}}=1 \mathrm{keV} ; \mathrm{B}=50 \mathrm{kG}$, $\mathrm{q}_{\mathrm{O}}=5 ; \varepsilon=1 / 3 ; \mathrm{a}=2.5 \mathrm{~m}$, one finds $\delta_{\mathrm{Cl}} \simeq 0.9 \times 10^{-2} \cdot \mathrm{A}$ mirror confined divertor plasma should have approximately the same width, for ions can only move a drift orbit before scattering into the loss cone. In principle, the width of a microturbulent scrapeoff. layer could be much wider. However, one would expect it to be no wider than predicted by Bohm diffusion. The Bohm law coupled with flow at the speed of sound along the field lines gives $\delta_{\mathrm{B}}^{2}=[\mathrm{Tc} /(16 \mathrm{eB})]\left[\mathrm{qR} /\left(\mathrm{T} / \mathrm{m}_{\mathrm{i}}\right)^{1 / 2}\right] / \mathrm{a}^{2}$. This can be rewritten as $\delta_{B}=\left(q r_{i} R / 16\right)^{1 / 2} / a$. With the same parameters as above, $\delta_{B} \simeq 2 \times 10^{-2}$.

Either classical or turbulent diffusion theory predicts a thin scrapeoff layer. It is important to determine if field errors will be the dominate widening mechanism. It is well known thal rield errors can destroy exact magnetic surfaces. $2-5$ 
magnetic perturbation with angúlar dependence $\exp [i(m \theta-n \phi)]$ produces a chain of islands (Fig. 2, read $m \theta$ for $\nu \theta_{*}$ ) about its resonant rational surface, $q=\mathrm{m} / \mathrm{n}$ with $\mathrm{q}$ the tokamak safety factor. Perturbations containing many harmonics produce a number of island chains. If the island chains overlap, the field lines become ergodic in the overlap region. In practice, this means a single field line will cover a finite volume instead of a surface. In this paper we will assume the field lines are ergodic in regions where the separation between island chains is. Iess than their width and that definite magnetic surfaces exist otherwise. This is, of course, a considerable simplification of the true situation. The demonstration that this assumption is nonetheless an adequate description of the process for practical purposes has been the subject of numerous papers and will not be considered here. 6

In the second section of this paper we will give a general method for finding the region of ergodic field lines about the separatrix magnetic surface (the bounding surface between. open and closed lines). The method is essentially that used by Rechester and stix to examine the ergodic region produced by a magnetic perturbation containing two poloidal mode (m) numbers. In the third section the general method will be applied to the simplest divertor-like geometry-the field of two infinite parallel wires. The results will be summarized in the final section. 


\section{GENERAL METHOD OF SOLUTION}

To simply evaluate the regions of ergodic field lines, an appropriate coordinate system is required. Conceptually, the simplest cóordinate system for a toroidally symmetric system is the $r, \theta, \phi$ system. The mathematics, however, is simplified by going to the related Hamada $\psi, \theta_{*}, \phi$ system. The new radial coordinate $\psi(r, \theta)$ is the flux associated with the poloidal magnetic field $\vec{B}_{p}=(\hat{\phi} / R) \times \vec{\nabla} \psi$. (The flux is closely related to the toroidal component of the vector potential of the magnetic field, $\left.\psi=-R A_{\phi}.\right)$ From the definition of $\psi$, it follows that $\overrightarrow{\mathrm{B}}_{\mathrm{O}} \cdot \vec{\nabla} \psi=0$ with $\overrightarrow{\mathrm{B}}_{\mathrm{O}}$ the unperturbed magnetic field. This means the unperturbed field lines lie in surfaces of constant $\psi$ called magnetic surfaces. A point on a magnetic surface is defined by the angles, $\theta$ and $\phi$. The angle $\theta$ may have only a limited range on some magnetic surfaces, say $|\theta| \leq \theta_{0}$ (sce Fig. 3 for an example). The angular coordinate $\theta_{*}$ is chosen so $\mathrm{d} \theta_{*} / \mathrm{d} \phi=1 / \mathrm{q}(\psi)$ with the total derivative implying differentiation along the magnetic field lines and with $q(\psi)$, the safety factor, being only a function $\psi$. The coordinate $\theta_{*}$ is also chosen so $\theta_{*}=0$ at $\theta=0$ and $\theta_{*}= \pm \pi$ at $\theta= \pm \theta_{0}$. Using the unperturbed magnetic field, specified by $\psi(r, \theta)$ and $\mathrm{B}_{\phi}(r, \theta)$ with $\mathrm{B}_{\theta}=(\partial \psi / \partial r) / \mathrm{R}$, one defines $Q(\psi, \theta) \equiv d \phi / d \theta=r B_{\phi} /\left(R B_{\theta}\right)$, then

$$
\frac{d \theta_{\star}}{d \theta}=\frac{d \theta_{\star}}{d \phi} \frac{d \phi}{d \theta}=\frac{Q(\psi, \theta)}{q(\psi)}
$$


This gives $\theta_{\text {* }}$ in a differential form (remember the total derivatives mean along a field line). The boundary conditions $\theta_{*}(\theta=0)=0$ and $\theta_{*}\left(\theta= \pm \theta_{0}\right)= \pm \pi$ imply

$$
\mathrm{q}(\psi)=\frac{1}{2 \pi} \int_{-\theta_{0}}^{\theta_{0}} \mathrm{Q}(\psi, \theta) \mathrm{d} \theta
$$

The integrations to evaluate $\theta_{\star}(\psi, \theta)$ and $q(\psi)$ are carried out at constant $\psi$ since they are to be along a field line. The symmetry direction angular coordinate $\phi$ is used in both coordinate systems. The physical significance of the $\psi, \theta_{*}, \phi$ coordinate system is the magnetic field has components only in the $\vec{\nabla} \theta_{\star}$ and $\vec{\nabla} \phi$ directions and the field lines wind about the magnetic axis at a constant pitch, $d \theta_{\star} / d \phi$, in a magnetic surface. It should be noted, "however, that in general $\vec{\nabla} \psi \cdot \vec{\nabla} \theta_{\star} \neq 0$; so the coordinate system $\psi, \theta_{\star}, \phi$ is not always orthogonal. For our analysis, this will not, create any difficulties.

We wish to solve the so-called magnetic differential equation $\vec{B} \cdot \vec{\nabla} \Phi=0$ for the function $\Phi$ with $\vec{B}$ the magnetic field including the perturbation $\vec{B}_{1} \therefore$ The magnetic field lines lie in surfaces of constant $\Phi$ (magnetic surfaces). If a function $\Phi$ can be found, which is monotonically increasing, then the field lines lie in nested toroidal surfaces. However, if there is a chain of magnetic islands centered about the flux surface $\psi_{0}$, then near this surface $\Phi$ is basically of the form 
$\Phi\left(\psi, \theta_{\star}, \phi\right) \div \Phi_{0}+\frac{1}{2}\left(\psi-\psi_{0}\right)^{2}-\left(\frac{\Delta}{2}\right)^{2} \sin \left[n\left(\phi-\phi_{0}\right)-v \theta_{*}\right]$

with $\mathrm{n}$ and: $\mathrm{U}$ integers and $\Phi_{0}, \phi_{0}$, and $\Delta$ constants. The perturbed magnetic surfaces are plotted in the $\psi, \theta_{*}, \phi$ coordinate system by inverting Eq. (3) to give $\psi$ as a function of $\theta_{\star}$ and $\phi$ for particular values of $\Phi$. That is

$\psi=\psi_{0} \pm\left\{2\left[\Phi-\Phi_{0}+\left(\frac{\Delta}{2}\right)^{2} \sin \left[n\left(\phi-\phi_{0}\right)-v \theta_{*}\right]\right\}^{1 / 2}\right.$.

For $\left[\Phi-\Phi_{0}\right]<(\Delta / 2)^{2}$ a solution to Eq. (4) exists only for a certain range of $\mathrm{n}\left(\phi-\phi_{0}\right)-v \theta_{*}$. This implies the magnetic surface lies in a finite angular region and is part of an island structure (Fig. 2). For $\left[\Phi-\Phi_{0}\right]>(\Delta / 2)^{2}$ the magnetic surface extends over the entire range of $n\left(\phi-\phi_{0}\right)-v \theta_{*}$ and encircles the axis of the unperturbed poloidal magnetic field. Surfaces which encircle the magnetic axis are topologically equivalent to the unperturbed surfaces. Indeed, for $\left[\Phi-\Phi_{0}\right]>(\Delta / 2)^{2}$, the perturbed surfaces are essentially identical to the unperturbed ones. The quantity $\Delta$ is the half width of the island in $\psi$ space. To see this we note that the separatrix magnetic surface between island magnetic surfaces and surfaces that exist for all angular positions is $\left[\Phi-\Phi_{0}\right]=(\Delta / 2)^{2}$. The equation for the separatrix surface is 
$\psi=\psi_{0} \pm \frac{\Delta}{2}\left(2\left\{1+\sin \left[n\left(\phi-\phi_{0}\right)-v \theta_{*}\right]\right\}\right)^{1 / 2}$

which demonstrates $\Delta$ is the half width of the islands. As pointed out in the introduction the field lines become ergodic when there are chains of islands about a number of $\psi$ surfaces and when the width of the islands $2 \Delta$ equals the separation in $\psi$ of the chains. In the magnetic differential equation, $\vec{B} \cdot \vec{\nabla} \Phi=0$, the magnetic field is the sum of an: unperturbed part $\vec{B}_{0}$ plus d pelturbed part $\vec{B}_{1}$. The function $\Phi$ is written as $\Phi=\Phi_{0}(\psi)+\Phi_{1}$, so $\vec{B}_{O} \cdot \vec{\nabla} \Phi_{0}=0$. The part $\Phi_{O}(\psi)$, is chosen to best approximate the solution so we can ignore the $\vec{B}_{1} \cdot \vec{\nabla} \Phi{ }_{1}$ term. For example, [Eq. (3)] for $\Phi$ near a chain of magnetic islands leads us to expect $d \Phi_{0} / d \psi=0$ for $\psi=\psi_{0}$ with the island centered about $\psi_{0}$. The equation we will solve is $\vec{B}_{0} \cdot \vec{\nabla} \Phi_{1}=-\left(\vec{B}_{1} \cdot \vec{\nabla} \psi\right)\left(\mathrm{d} \Phi_{0} / \mathrm{d} \psi\right)$. Now $\Phi_{1}$ is a function of $\psi, \theta_{*}, \phi$ so

$\overrightarrow{\mathrm{B}}_{0} \cdot \vec{\nabla} \Phi_{1}=\left(\overrightarrow{\mathrm{B}}_{0} \cdot \vec{\nabla} \psi\right)\left(\frac{\partial \Phi_{1}}{\partial \psi}\right)+\left(\overrightarrow{\mathrm{B}}_{0} \cdot \vec{\nabla} \theta_{\star}\right) \cdot\left(\frac{\partial \Phi_{1}}{\partial \theta_{*}}\right)+\left(\overrightarrow{\mathrm{B}}_{0} \cdot \vec{\nabla} \phi\right)\left(\frac{\partial \Phi_{1}}{\partial \phi}\right)$

$$
=\frac{\mathrm{B}_{\phi}}{\mathrm{R}}\left(\frac{\partial \Phi}{\partial \phi}+\frac{1}{\mathrm{q}} \frac{\partial \Phi_{1}}{\partial \theta_{\star}}\right)
$$


where we have used $\overrightarrow{\mathrm{B}}_{\mathrm{O}} \cdot \vec{\nabla} \psi=0,\left(\overrightarrow{\mathrm{B}}_{\mathrm{O}} \cdot \vec{\nabla} \theta_{\star}\right) /\left(\overrightarrow{\mathrm{B}}_{\mathrm{O}} \cdot \vec{\nabla} \phi\right)=\mathrm{d} \theta_{\star} / \mathrm{d} \phi=1 / \mathrm{q}(\psi)$, and $\vec{B}_{O} \cdot \vec{\nabla}_{\phi}=B_{\phi} / R$. The perturbation is written in the form

$$
\overrightarrow{\mathrm{B}}_{1} \cdot \vec{\nabla}_{\psi}=\frac{\mathrm{B}_{\phi}}{\mathrm{R}} \mathrm{b}\left(\psi, \theta_{\star}\right) \mathrm{e}^{\mathrm{in} \phi}
$$

with a definite toroidal mode number $\mathrm{n}$ assumed. The magnetic differential equation can now be given as

$$
\frac{\partial \Phi}{\partial \phi}+\frac{1}{q(\psi)} \frac{\partial \Phi_{1}}{\partial \theta_{\star}}=-\frac{d \Phi}{d \psi} b\left(\psi, \theta_{\star}\right) e^{i n \phi}
$$

This equation is simply solved by a Fourier transform. Define $\Phi_{v}(\psi)$ and $\mathrm{b}_{v}(\psi)$ so

$$
\Phi_{1}=\sum_{\nu=-\infty}^{\infty} \Phi_{\nu} \dot{\exp }\left[\mathrm{i}\left(\mathrm{n} \phi-\nu \theta_{\star}\right)\right], \mathrm{b}_{\nu}=\frac{1}{2 \pi} \int_{-\pi}^{\pi} \mathrm{b}\left(\psi, \theta_{\star}\right) \exp \left(i v \theta_{\star}\right) d \theta_{*}
$$

and one obtains the algebraic equation for $\Phi_{V}(\psi)$

$$
i\left(n-\frac{v}{q}\right) \Phi_{v}=-b_{v} \frac{d \Phi_{0}}{d \eta}
$$


This equation is singular for $\mathrm{nq}(\psi)$ equal to an integer unless $\mathrm{d} \Phi / \mathrm{d} \psi$ vanishes. The interpretation is that there is a chain of islands about $\psi=\psi_{\nu}$ with $n q\left(\psi_{\nu}\right)=\nu$ where $\nu$ is any integer. Letting $q^{\prime}=d q / d \psi$ we can easily form a $\Phi_{0}(\psi)$ which has a zero derivative at $\psi=\psi_{\nu} \cdot$ we let

$$
\frac{d \Phi}{d \psi}=\frac{l}{\pi n q} \sin (\pi n q)
$$

which can be integrated. to form $\Phi_{0}(\psi)$. Using this expression for $\mathrm{d} \Phi_{\mathrm{o}} / \mathrm{d} \psi$

$$
\Phi_{v}(\psi)=i \frac{q}{q} \frac{b_{v}}{n \pi} \frac{\sin (\pi n q)}{n q-v} \therefore
$$

If the chains of islands are well separated, we can look at the solution near each chain independently. For

$$
\begin{aligned}
& \left|\psi-\psi_{v}\right|<\left|\psi_{v}-\psi_{v+1}\right|, \Phi_{v} \simeq \dot{i}\left(q / q^{\prime}\right)\left(b_{v} / n\right) \text { and } \Phi \text { is } \\
& \Phi=\dot{\Phi}_{0}\left(\psi_{\nu}\right) \pm\left(\frac{1}{2}\left(\psi-\psi_{v}\right)^{2}-\left|\frac{1}{n} \frac{\mathrm{q}}{\mathrm{q}} \mathrm{b}_{v}\right| \sin \left[\mathrm{n}\left(\phi-\phi_{0}\right)-v \theta_{*}\right]\right) \text {. }
\end{aligned}
$$


The half width $\Delta_{\nu}$ of islands near $\psi_{\nu}$ is given by [compare with Eqs. (4) and (5)]

$$
\left(\frac{\Delta_{v}}{2}\right)^{2}=\left|\frac{1}{n} \frac{q}{q} b_{v}\right|
$$

This approximate expression for the island width $2 \Delta_{v}$ can be used to predict the overlap of chains of islands and hence ergodicity. The distance in $\psi$ space between chains of islands is $\psi_{v+1}-\psi_{\nu}$ with $n q\left(\psi_{v+1}\right)=1+n q\left(\psi_{v}\right)$, or $\psi_{v+1}-\psi_{v} \simeq 1 / n q$ ' Equating the separation to the width of the islands, one has $2 \Delta_{v}=1 /(n q$ ') as the condition for overlap. Combining this result for our approximate expression for $\Delta_{v}$, we have the condition for ergodicity

$\left|16 \mathrm{nq} \frac{\mathrm{dq}}{\mathrm{d} \psi} \mathrm{b}_{\nu}\right|>1$ with $v=\mathrm{nq}(\psi), \mathrm{b}_{\nu}=\frac{1}{2 \pi} \int_{-\pi}^{\pi} \frac{\mathrm{R}}{\overrightarrow{\mathrm{B}}_{\phi}}\left(\overrightarrow{\mathrm{B}}_{10} \cdot \vec{\nabla} \psi\right) \exp \left(\mathrm{i} v \theta_{*}\right) \mathrm{d} \theta_{*} \cdot$

The perturbation magnetic field $\vec{B}_{1}$ has been written $\vec{B}_{1}=$ $\vec{B}_{10} \exp (i n \phi)$. At the separatrix between the open field lines of the scrapeoff region and the closed field lines of the interior, one can show (see next section) $q(\psi) b_{\nu}$ is finite while dq/d $\psi$ is singular. This implies the condition for ergodicity is always satisfied close enough to the separatrix magnetic surface. In the next section we will evaluate the width of this ergodic layer in a simple magnetic configuration. 


\section{ANALYTIC EXAMPLE}

To illustrate the method outlined in the last section, we will consider the magnetic field produced by equal currents in two infinite parallel wires (Fig. 3). The width of the region of ergodic field lines about the separatrix will be studied qualitativoly fọr a general perturbation and evaluated exactly for the toroidal ripple perturbation with the minimum of the ripple at the $x$ point. This system is not toroidal, hut the toroidal formalism can be used by letting $\phi=z / R$ and assuming the perturbed system is periodic in $\mathrm{z}$ with a period $2 \pi \mathrm{R}$. The distance scale: $\mathrm{R}$ can otherwise be considered arbitrary.

The first part of the problem is to formulate mathematically the magnetic field structure and establish the $\psi, \theta_{*}, \phi$ coordinate system. The unperturbed magnetic field is $\overrightarrow{\mathrm{B}}_{0}=\mathrm{B}_{\phi} \hat{\psi}+(\hat{\phi} / \mathrm{R}) \times \vec{\nabla} \psi$. Letting $\mathrm{r}_{+}$be the radius vector about. $x=a, y=0$, the flux $\psi_{+}$due to the current $I$ in the wire at that point is

$$
\psi_{+}=R \int_{a}^{r_{+}} \frac{2 I}{r_{+}} d r_{+}=\frac{2}{c} \operatorname{IR} \text { en }\left(\frac{r_{+}}{a}\right)
$$

A similar expression for $\psi_{-}$, the flux due to the current I in the wire at $x=-a, y=0$, can be obtained. The total flux is $\psi=\psi_{+}+\psi_{-}$so 


$$
\psi=\frac{2}{c} \operatorname{IR} \ln \left[\left(\frac{{ }^{r}}{\mathrm{a}}\right)\left(\frac{\mathrm{r}-}{\mathrm{a}}\right)\right]=\mathrm{aRB}_{\mathrm{p}} \ln (\mathrm{p})
$$

with $\mathrm{B}_{\mathrm{p}}=2 \mathrm{I} /(\mathrm{ca})$, the magnitude of the poloidal field at the $x$ point due to one wire, and the dimensionless parameter $p$ defined by $\mathrm{p}=\left(r_{+} / \mathrm{a}\right)\left(r_{-} / a\right)$. Inspection shows that $\mathrm{p}<1$ gives magnetic surfaces inside the separatrix and $\mathrm{p}>1$ outside. For this problem $\mathrm{p}$ is a more convenient coordinate than $\psi$ and clearly equivalent to it. Consequently, the $p, \theta_{*}, \phi$ coordinate system will be used. The simplest initial coordinate system is the cylindrical system $r, \theta, \phi$ centered on the $\mathrm{x}$ point. In these coordinates

$$
\mathrm{p}^{2}=\left(\frac{r}{\mathrm{a}}\right)^{4}-2\left(\frac{r}{\mathrm{a}}\right)^{2} \cos (2 \theta)+1 .
$$

The coordinate transformation goes exactly as outlined in the last section. One has $d \theta_{*} / d \theta=\Omega(p, \theta) / q(p)$ with $q(p)$ the safety factor. The angle $\theta_{*}$ advances by $2 \pi$ as it circles the magnetic axis. Using 


$$
\mathrm{B}_{\theta}=\frac{1}{\mathrm{R}} \frac{\partial \psi}{\partial \mathrm{r}}=\frac{2 \mathrm{~B}}{\mathrm{p}^{2}}\left[\left(\frac{r}{\mathrm{a}}\right)^{3}-\left(\frac{r}{\mathrm{a}}\right) \cos (2 \hat{\theta})\right]
$$

the quantity $Q(p, \theta)=r B_{\phi} /\left(R B_{\theta}\right)$. The radius $r$ is replaced by $\mathrm{p}$ and $\theta$ [using $\mathrm{Eg} \cdot(18)]$ in the equation for $\mathrm{Q}$. The evaluativin of $\theta_{*}$ can involve unfamiliar algebra for the final result must be expressed in terms of the Jacobi clliptis functions $\operatorname{sn}(u), \operatorname{dn}(u)$, and $\operatorname{cn}(u)$.

To understand the form of $\theta_{*}$ and to be able to check the results, a few properties of the Jacobi elliptic functions are required. These functions obey the identities $\mathrm{cn}^{2}(u)+\mathrm{sn}^{2}(u)=1$, $\mathrm{dn}^{2}(u)+k^{2} \operatorname{sn}^{2} u=1$, and $d \operatorname{sn}(u) / d u=c n(u) d n(u)$. The parameter $k$ is the so-called index of the elliptic functions, $0 \leq k: \leq 1$. For $k=0, \operatorname{sn}(u)=\sin (u), \operatorname{cn}(u)=\cos (u)$, and $\operatorname{dn}(u)=1$. For $k=1, \operatorname{sn}(u)=\tanh (u) \operatorname{and} \operatorname{cn}(u)=\operatorname{dn}(u)=\operatorname{sech}(u) \cdot \operatorname{Bọth}$ sn $(u)$ and $c n(u)$ have real periods of $4 \mathrm{~K}$ and $\mathrm{dn}(\mathrm{u})$ a real period of $2 \mathrm{~K}$ with $\mathrm{K}(\mathrm{k})$ the completc elliptic integral of the first kind. For $k \rightarrow 0, K(k)=\pi / 2$ while for $k \rightarrow 1, K(k)=\ln [8 /(1-k)]^{1 / 2}$. It is also customary to define the complementary index $k$ ' by $\mathrm{k}^{2}+\mathrm{k}^{\prime 2}=1$ and $\mathrm{K}^{\prime}$ by $\mathrm{K}^{\prime}=\mathrm{k}\left(\mathrm{k}_{i}^{\prime}\right)$.

The transformation from the cylindrical coordinate system $r, \theta$ to the $\mathrm{p}, \theta_{*}$ one can now be given. Letting $u=(4 \mathrm{~K} / 2 \pi) \theta_{*}$ For $\mathrm{p}<1, \sin (2 \theta)=\mathrm{p} \sin (\mathrm{u}),\left(\frac{\mathrm{r}}{\mathrm{a}}\right)^{2}=\mathrm{dn}(\mathrm{u})+\mathrm{p} \operatorname{cn} u, \mathrm{k}=\mathrm{p}$ For $p>1, \sin (20)=\operatorname{sn}(2 u),\left(\frac{r}{a}\right)^{2}=p d n(2 u)+\operatorname{cn}(2 u) \cdot k=\frac{1}{p}$. 
The quadrant conventions to be used are given by Fig. 4. The safety factor $q(p)$ can be evaluated using $q(p)=d \phi / d \theta_{+}=$ $(4 \mathrm{~K} / 2 \pi)(\mathrm{d} \theta / \mathrm{du})(\mathrm{d} \phi / \mathrm{d} \theta)$ and differentiating Eq. (20) at constant $p$ to obtaini $d \theta / d u$ and noting that $d \phi / d \theta=r B_{\phi} / R B_{\theta} \cdot$ Defining $q_{O}=\left(a B_{\phi}\right) /\left(R B_{p}\right)$, the answer is

For $p<1, q(p)=\frac{q_{O} p^{2}}{2 \pi} k(p)$
For $p>1, q(p)=\frac{q_{O} p}{\pi} k\left(\frac{1}{p}\right)$.

In the limit $p>1$, one can show $\theta_{*}=\theta, r / a=p^{1 / 2}$, and $q=\left(a_{O} / 2\right)(r / a)^{2}$. This is the $q$ of a current of strength $2 I$ at a radius $r$ as one would expect. In the limit $p<1$, there are two solutions depending on which wire one is closer to. In the region near the wire at $x=a, y=0$ (see Fig. 3), $\mathrm{p}=2 \mathrm{r}_{+} / \mathrm{a}$ and $\theta_{\star}=\theta_{+}$with $r_{+}, \theta_{+}$cylindrical coordinates centered at $x=a, y=0$. The $q$ value is $q=q_{0}\left(r_{+} / a\right)^{2}$ as one would expect.

With the $p, \theta_{*}, \phi$ coordinate system established, the implications of the ergodicity condition [Eq. (15)] can be studied. Since the ergodic region is expected to be near the separatrix, the equation will be examined for $p$ near but less than one. In this limit the equation for $b_{v}$ can be written using $u=(4 K / 2 \pi) \theta_{*}$ as 


$$
b_{v}=\frac{1}{4 K} \int_{-\infty}^{\infty} \frac{R}{B_{\phi}} \vec{B}_{10} \cdot \vec{\nabla} p \operatorname{expi} \lambda u d u
$$

with $\lambda=(2 \pi / 4 \mathrm{~K}) \cup$. Letting $\delta=1-\mathrm{p}$ and noting that $\mathrm{dq} / \mathrm{dp}=$ $q_{0} /(4 \pi \delta)$, the inequality of Eq. (15) implies ergodicity if

$$
\delta \leq \frac{n}{2 \pi^{2}} q_{0}^{2}\left|\int_{-\infty}^{\infty} \frac{R}{B_{\phi}} \vec{B}_{10} \cdot \vec{\nabla}_{p} \operatorname{cxp}(j, \lambda u) \pi_{11}\right|
$$

Since $v=n q$ in Eq. (15), one finds $\lambda=n_{0} / 4$. The determining factor in: the width of the ergodic region $\delta$ is clearly the Fourier integral. The integrand consists of three factors. First, there is the term $\left(R / B_{\phi}\right) \vec{B}_{10} \cdot \vec{\nabla} \mathrm{p} /|\vec{\nabla} \mathrm{p}|$. This term is the perturbation magnetic field orthogonal to the original magnetic surface normalized by the toroidal field and the aspect ratio. Second, there is a term $|\vec{\nabla} \mathrm{p}|=2 \mathrm{r} / \mathrm{a}^{2}=2 \sqrt{2}(\operatorname{sech} \mathrm{u})^{1 / 2} / \mathrm{a}$ which essentially is the strength of the poloidal field. This term vanishes exponentially for large $u$. Consequently, only the value of the perturbation well away from the $x$ point is relevant. Finally, there is the oscillating exponential $\exp (i \lambda u)$ which gives resonance effects. The maximum $\delta$ which can occur is found by letting $\left(\vec{B}_{10} \cdot \vec{\nabla} \mathrm{p} /|\vec{\nabla} \mathrm{p}|\right)_{\text {MAX }}=\mathrm{B}_{\perp},|\vec{\nabla} \mathrm{p}|=2 \sqrt{2}(\operatorname{sech} u)^{1 / 2} / \mathrm{a}, \exp (i \lambda u)=1$, that is 


$$
\delta_{\mathrm{MAX}}=\frac{\sqrt{2}}{\pi^{2}}\left(\frac{[\Gamma(1 / 4)]^{2}}{\sqrt{2} \Gamma(1 / 2)}\right) \mathrm{nq}_{\mathrm{O}} \frac{\mathrm{B}_{\perp}}{\mathrm{B}_{\mathrm{p}}} \simeq 0.7514 \mathrm{nq}_{\mathrm{O}} \frac{\mathrm{B}_{\perp}}{\mathrm{B}_{\mathrm{p}}}
$$

The expression in parenthesis is the integral of $(\operatorname{sech} u)^{1 / 2}$ from minus to plus infinity. The maximum width for the ergodic region $\delta_{\text {MAX }}$ only occurs when there is a resonance between the pcrturluation and the exponential $\exp (i \lambda u)$. One can represent this effect by letting $\vec{B}_{10} \cdot \vec{\nabla} \mathrm{p} /|\vec{\nabla} \mathrm{p}|=\mathrm{B}_{\perp} \exp \left(\operatorname{im} \theta_{+}\right)$which $\theta_{+}$the angular coordinate about the wire at $x=a, y=0$. One finds that for $u<1, \theta_{f}$ and $u$ are essentially proportional with $\theta_{+} / u \simeq 1 /(2-\sqrt{2})=1.7071$ (see Fig. 5). Since the region $\mathrm{u}<1$ dominates the integral for $\delta$, we can approximate $m \theta_{+}$by $\mathrm{m}^{\prime} \mathrm{u}$ with $\mathrm{m}^{\prime}=1.7071 \mathrm{~m}$. The required integral can be performed in terms of the complex gamma functions. An approximation which gives the basic behavior is

$$
\delta \approx \frac{3}{4} \text { nq. }_{\mathrm{o}} \frac{\mathrm{B}_{\perp}}{\mathrm{B}_{\mathrm{p}}} \exp \left(-\frac{\pi}{2}\left|\lambda-\mathrm{m}^{\prime}\right|\right)
$$

The factor in the exponential can be written in a more transparent manner with $\pi\left(\lambda-\mathrm{m}^{\prime}\right) / 2 \simeq 2.68\left(\mathrm{nq}_{\mathrm{R}}-\mathrm{m}\right)$ with $\mathrm{q}_{\mathrm{R}} \equiv \mathrm{q}_{\mathrm{O}} / 6.83$. To make these results physical, the relationship between $\delta$ and the width of the ergodic region in centimeters is required. Letting $\delta=|1-p|$ one finds using Eq. (18), the width of the 
ergodic region near the $x$ point is $a \delta^{1 / 2}$ with $2 a$ the distance between the wires. At the furthest point on the separatrix magnetic surface from the $\mathrm{x}$, point, the ergodic region width is a $\delta / 2^{3 / 2}$. The delta of the introduction is the same as the delta here; so the dividing line between ergodicity being important and irrelevant is $\delta \sim 10^{-2}$.

The approximatinns wlitih went into Fig. (25) for $\delta$ are crude enough that an exact solution is of considerable importalle. The simplest perturbation to analyze analytically 15 tur idal ripple with the minimum of the ripple at the $x$ point. The perturbation is

$$
\vec{B}_{1}=\frac{B_{R}}{I_{0}(n \sqrt{2} a / R)}\left[\hat{r} I_{1}\left(\frac{n r}{R}\right) \cos n \phi-\hat{\phi} I_{0}\left(\frac{n r}{R}\right) \sin n \phi\right]
$$

with $I_{0}$ and $I_{1}$ the modified Bessel functions. This perturbation satisfies the equations $\vec{\nabla} \cdot \vec{B}_{I}=0$ and $\vec{\nabla} \times \vec{B}_{I}=0$. The normalization is chosen so the maximum ripple felt on a magnetic surface is $B_{R}$. Using Eq. (7) to define $b\left(p, \theta_{+}\right)$we have

$$
\mathrm{b}=\frac{2}{\mathrm{nI}_{\mathrm{O}}(\mathrm{n} \sqrt{2} \mathrm{a} / \mathrm{R})}\left(\frac{\mathrm{R}}{\mathrm{a}}\right)^{2} \frac{\mathrm{B}_{\mathrm{R}}}{\mathrm{B}_{\mathrm{T}}} \frac{\mathrm{nr}}{\mathrm{R}} i_{1}\left(\frac{\mathrm{nr}}{\mathrm{R}}\right)\left\{\begin{array}{c}
\mathrm{cn} \mathrm{u}, \mathrm{p}<1 \\
\vdots \\
\mathrm{dn} 2 \mathrm{u}, \mathrm{p}>1
\end{array}\right.
$$


Of course, $r$ must be expressed in terms of $p$ and $u$ using Eq. (20). Mathematically, the messiest part of the calculation is the Fourier transformation in $\theta_{*}$ of $b\left(p, \theta_{*}\right)$ to obtain $b_{v}(p)$ : To carry out the integrals, we found it necessary to Taylor expand $I_{1}(x)$ and then resum the series at the end of the calculation.

$$
I_{I}(x)=\sum_{\ell=0}^{\infty} \frac{1}{\ell !(\ell+1) !}\left(\frac{x}{2}\right)^{2 \ell+1} .
$$

Two integrals are defined with $\lambda=2 \pi \nu / 4 \mathrm{~K}$

$$
\begin{aligned}
& g_{\ell}(\lambda)=\int_{0}^{4 K}[\operatorname{dn}(u)+k \operatorname{cn}(u)]^{\ell+1} \operatorname{cn}(u) \exp (i \lambda u) d u \\
& h_{\ell}(\lambda)=\int_{0}^{4 K}\left(\frac{1}{k} \operatorname{dn}(2 u)+\operatorname{cn}(2 u)\right)^{\ell+1} \operatorname{dn}(2 u) \exp (i \lambda u) d u
\end{aligned}
$$

then one finds using $u=4 K \theta_{*} / 2 \pi$

$$
\begin{aligned}
& b_{v}=\frac{1}{2 \pi} \cdot \int_{0}^{2 \pi} b e^{i \nu \theta_{*}} d \theta_{*}
\end{aligned}
$$

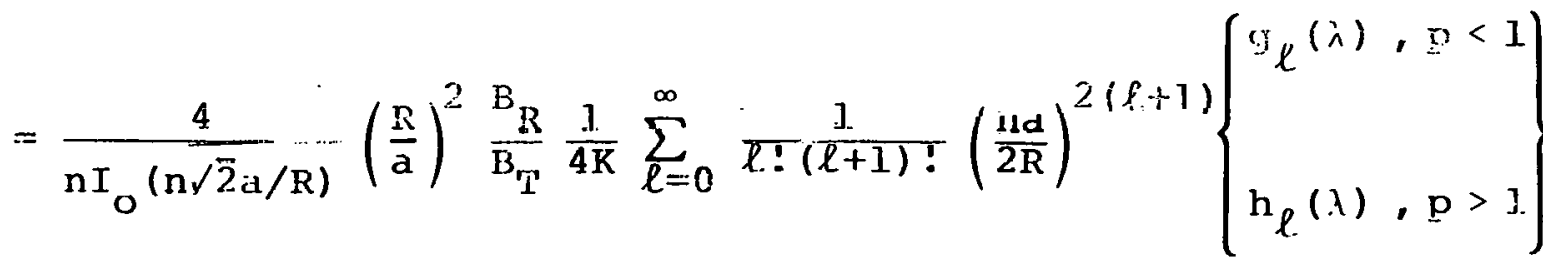


The integrals $g_{\ell}$ and $h_{\ell}$ are performed in Appendix $A ;$ the results are

$$
g_{\ell \cdot}(\lambda)=\frac{\pi}{\mathrm{k}} \frac{(2 \lambda)^{\ell+1}}{(l+1) !}\left\{\begin{array}{l}
\operatorname{cosech}\left(\mathrm{K}^{\prime} \lambda\right), l \text { even } \\
\operatorname{sech}\left(\mathrm{K}^{\prime} \lambda\right), \ell \text { odd }
\end{array}\right\}
$$

with $K^{\prime}$ the complete elliptic integral of the first kind of complimentary argument, $\mathrm{K}^{\prime}=\mathrm{K}\left(\mathrm{k}^{\prime}\right), \mathrm{k}^{2}+\mathrm{k}^{\prime}=1$. The integrail $\mathrm{h}_{\ell}(\lambda)$ is zero for $\nu$ odd and $h_{\ell}(\lambda)=, \mathrm{k}^{-\ell} g_{\ell^{(}}(\lambda / 2)$ for $\nu$ even. A function $\mathrm{H}(\mathrm{x})$ is defined along with its even, $\mathrm{H}_{\mathrm{e}}(\mathrm{x})$, and odd, $\mathrm{H}_{\mathrm{O}}(\mathrm{x})$, parts

$H(x)=\sum_{\ell=1}^{\infty} \frac{x^{\ell}}{(\ell-1) !(\ell !)^{2}}, H_{e}(x)=\frac{H(x)+H(-x)}{2}, H_{0}(x)=\frac{H(x)-H(-x)}{2}$.

Letting $\beta=n a / R, \lambda=2 \pi v / 4 \mathrm{~K}$ the Fourier transform of the normalized perturbation is

$\mathrm{p}<1, \mathrm{~b}_{\nu}=\left(\frac{\mathrm{R}}{\mathrm{a}}\right)^{2} \frac{\mathrm{B}_{\mathrm{R}}}{\mathrm{B}_{\mathrm{T}}} \frac{\pi}{\operatorname{npK} I_{\mathrm{O}}\left(\mathrm{n} \sqrt{2} \mathrm{a}_{j} \mathrm{R}\right)}\left(\frac{\mathrm{H}_{\mathrm{O}}\left(\beta^{2} \lambda / 2\right)}{\sinh \left(\mathrm{K}^{\prime} \lambda\right)}+\frac{\mathrm{H}_{\mathrm{e}}\left(\beta^{2} \lambda / 2\right)}{\cosh \left(\mathrm{K}^{\prime} \lambda\right)}\right)$

$\mathrm{p}>1, v$ even, $\mathrm{b}_{\nu}=\left(\frac{\mathrm{R}}{\mathrm{a}}\right)^{2} \frac{\mathrm{B}_{\mathrm{B}}}{\mathrm{B}_{\mathrm{T}}} \frac{\pi}{\mathrm{nK} \mathrm{I}_{\mathrm{O}}(\mathrm{n} \sqrt{2} \mathrm{a} / \mathrm{R})}\left(\frac{\mathrm{H}_{\mathrm{O}}\left(\mathrm{p} \beta^{2} \lambda / 4\right)}{\sinh \left(\mathrm{K}^{\top} \lambda / 2\right)}+\frac{\mathrm{H}_{\mathrm{e}}\left(\mathrm{p} \beta^{2} \lambda \gamma / 4\right)}{\cosh \left(\mathrm{K}^{\prime} \lambda / 2\right)}\right)$

$\mathrm{p}>1, v$ odd, $\mathrm{b}_{v}=0$. 
The expression for $b_{v}$ is to be inserted in Eq. (15) to give the ergodic region. There is a minor complication for $p>1$. In deriving Eq. (15), we assumed $\left|b_{v}\right|>0$ for all $v$. Due to the high symmetry of our geometry, $b\left(p, \theta_{*}+\pi\right)=b\left(p, \theta_{*}\right)$ for $\mathrm{p}>1$, the odd Fourier terms vanish identically. The necessary correction to Eq. (15) is simple--the factor of 16 becomes 4 for $\mathrm{p}>1$

For small perturbations, the ergodic region lies close to the separatrix surfaces. Consequently, p can be assumed near unity while evaluating the width. When $p$ is near one, so is the index of the elliptic functions $k$. The asymptotic forms $(k \rightarrow 1)$ that are required to evaluate the width of the ergodic region are $\mathrm{K}^{\prime}=\pi / 2$ and $2 \mathrm{dK} / \mathrm{dk}=1 /(1-\mathrm{k})$. When it is narrow, the ergodic region is centered about the separatrix surface with the range of $p$ which lies in the ergodic zone given by

$$
\begin{aligned}
& |l-p| \leq \delta \\
& \delta=\frac{2}{\pi I_{0}(n \sqrt{2} a / R)}\left(\frac{q_{0}^{R}}{a}\right)^{2} \frac{B_{R}}{B_{T}}\left(\frac{H_{0}\left[n^{3}(a / R)^{2} q_{O} / 8\right]}{\sinh \left(\pi n q_{O} / 8\right)}+\frac{\mathrm{H}^{\left[n^{3}(a / R)^{2} q_{0} / 8\right]}}{\cosh \left(\pi_{\left.n q_{O} / 8\right)}\right.}\right)
\end{aligned}
$$

The expression for $\delta$, the half width of the ergodic region, is sufficiently messy that limiting forms are of value. For small toroidal mode numbers $n$,

$$
\delta \simeq \frac{1}{4 \pi} \frac{\mathrm{B}_{\mathrm{R}}}{\mathrm{B}_{\mathrm{T}}} \frac{\left(\mathrm{nq}_{\odot}\right)^{3}}{\sinh \left(\pi n \mathrm{q}_{\mathrm{O}} / 8\right)}, \mathrm{n}^{3} \ll \frac{8}{\mathrm{q}_{\mathrm{O}}}\left(\frac{\mathrm{R}}{\mathrm{a}}\right)^{2}, \mathrm{n} \ll \mathrm{R} / \mathrm{a}
$$


The opposite limit, large toroidal mode number $n$, exhibits a somewhat more complicated form. To examine this limit, the asymptotic forms of $\mathrm{H}(\mathrm{x})$ (derived in Appendix $\mathrm{B}$ ) and $\mathrm{I}_{\mathrm{O}}(\mathrm{x}$ ) are required for large $x$. These are

$$
\mathrm{II}(x \rightarrow \infty)=\frac{1}{2 \pi \sqrt{3}} \operatorname{crp}\left(3 \mathrm{x}^{1 / 3}\right), I_{0}(x \rightarrow \infty)=\frac{\exp x}{(2 \pi x)^{1 / 2}}
$$

The even and odd parts of $H(x)$ are each equal $H(x) / 2$ in the limit of large $x$. Assuming $(\pi / 8) n_{0}>1$, one finds

$$
\begin{aligned}
& \delta=\frac{2^{7 / 4}}{\left(3 \pi^{3}\right)^{1 / 2}} \frac{\mathrm{nq}_{\mathrm{O}}}{(\mathrm{na} / \mathrm{R})^{1 / 2}} \frac{\mathrm{B}_{\mathrm{R}}}{\mathrm{B}_{\mathrm{p}}} \exp \left(-\frac{\pi}{8} \mathrm{nq}_{\mathrm{O}}-\mathrm{n} \frac{\mathrm{a}}{\mathrm{R}}\left[\sqrt{2}-\frac{3}{2}\left(\mathrm{q}_{0} \frac{\mathrm{R}}{\mathrm{a}}\right)^{1 / 3}\right]\right) \\
& \mathrm{n}^{3}>\frac{8}{\mathrm{q}_{\mathrm{O}}}\left(\frac{\mathrm{R}}{\mathrm{a}}\right)^{2}, \mathrm{n}>\frac{\mathrm{R}}{\mathrm{a}} .
\end{aligned}
$$

The limiting forms for $\delta$ behave qualitatively like the approximate expression $\mathrm{Eq}$. (25). With a clever estimate for $\mathrm{B}_{\perp}$ one can obtain quantitative agreement. 
IV. SUMMARY

A general method has been developed for finding the width of the scrape-off layer of a tokamak due to magnetic perturbations. The unperturbed magnetic field is described by a Hamada $\psi, \theta_{*}, \phi$ coordinate system. In this coordinate system $\phi$ is the toroidal angle, $\hat{\phi} \times(\vec{\nabla} \psi) / R$ is the poloidal field, and $d \theta_{\star} / d \phi=1 / q(\psi)$ with $q(\psi)$ the safety factor (the total derivative implies differentiation along a field line). Using this coordinate system, a region of ergodic field lines is found near the separatrix surface. The condition that a particular rational magnetic surface (with $\mathrm{q}=\nu / \mathrm{n}, \nu$ and $\mathrm{n}$ integers) lie in the ergodic region is given by Eq. (15). In deriving this equation, we have assumed the perturbing magnetic field is of the form $\vec{B}_{1}=\vec{B}_{10} \exp (\operatorname{in} \phi)$. Given a definite unperturbed magnetic field for a tokamak with a divertor and a particular scrape-off width due to field errors which is desired, Eq. (15) determines the magnitude of the perturbation with a given spacial behavior which satisfies these requirements. Since the scrape-off width produced by a perturbation depends only on the Fourier transform of the $\theta_{*}$ coordinate of the component of the perturbation orthogonal to the magnetic surface, the analysis can be done quite rapidly on a computer. The simplest divertor-like system is two parallel wires carrying equal currents and separated by a distance $2 a$ (Fig. 3). In studying this system, it is convenient to introduce a 
dimensionless parameter $\delta$, as in the introduction, so the width of the ergodic region is a $\delta^{1 / 2}$ near the $x$ point and $a \delta / 2^{3 / 2}$ at the farthest point on the separatrix from the $x$ point. A qualitatively correct expression for $\delta$ due to a perturbation with poloidal. and toroidal mode numbers $m$ and $n$ relative to the magnetic axis of the plasma is

$$
\delta \approx \frac{3}{4}: n_{\circ} \frac{\mathrm{R}_{1}}{\mathrm{~B}_{\mathrm{p}}} \exp \left(-\frac{\pi}{2}\left|\frac{\mathrm{nq}_{\mathrm{o}}}{4}-\frac{\mathrm{m}}{2-\sqrt{2}}\right|\right) \text {. }
$$

$B_{\perp}$ is an average value of the perturbation orthogonal to the separatrix magnetic surface. $\quad B_{p}$ and $q_{o}$ are the poloidal field and $q$ value which would occur at the $x$ point if the wire representing the divertor coil were turned off. The factor in the exponential can be written $2.6 \dot{8}\left|\mathrm{nq}_{\mathrm{R}}-\mathrm{m}\right|$ with $\mathrm{q}_{\mathrm{R}}=\mathrm{q}_{\mathrm{O}} / 6.83$. The width of the ergodic layer has a resonant structure with $q_{R}$ giving the resonant values of $m$ and $n$. In practice one would expect $q_{R}$ to have a value close to unity. A rigorous upper limit exists on $\delta$ in this geometry. Letting $B_{\perp}$ be the maximum value of the perturbation perpendicular to the separatrix surface, one finds $\delta \leq 0.75 \mathrm{nq}_{0} \mathrm{~B}_{\perp} / \mathrm{B}_{\mathrm{p}}$. In addition to the qualitative results, an exact calculation was carried for the ergodic width due to the toroidal rippie perturbation with the minimum of the ripple at the $x$ points. The results of this calculation are given in Eqs. (33) to (36). It should be stressed that the 
ergodic layer thickness depends on the strength of the component of the magnetic perturbation orthogonal to the original surfaces. The thickness of the ergodic layer is insensitive to the strength of the perturbation in the vicinity of the $x$ point; the major contributions come from that part of the separatrix surface well away from the $x$ point. In present day tokamaks, magnetic perturbations are present with all small $\mathrm{m}$ and $\dot{\mathrm{n}}$ numbers with a strength of a few times $10^{-3}$ of the poloidal field. ${ }^{7}$ one might expect these perturbations to give a $\delta$ in the range of $10^{-2}$ to $10^{-3}$ which is close though possibly smaller than the effects discussed in the introduction. Toroidal ripple effects $(n>12)$ would appear to be small due to their nonresonant nature.

\section{ACKNOWLEDGMENT}

This work was supported by United States Energy Research and Development Administration Contract No. E(11-1)-3073. 


\section{APPENDIX A}

The following integrals need to be performed for $l \geq 0$, $\lambda=2 \pi \nu / 4 \mathrm{~K}$ with $\nu$ an integer.

$g_{\ell}(\lambda)=\int_{n}^{4 K} G_{\ell}(u) \exp (i \lambda u) d u, G_{\ell}(u)=(d n u+k \text { cn } u)^{\ell+1}$ cn $u$ $h_{\ell^{(}}(\lambda)=\int_{0}^{4 K} H_{\ell}(2 u) \exp (i \lambda u) d u, H_{\ell}(2 u)=\left(\frac{1}{k} \text { dn } 2 u+\operatorname{cn} 2 u\right)^{\ell+1}$ dn $2 u$.

The second integral can be put in an easier form to integrate by using the $4 \mathrm{~K}$ periodicity of $\mathrm{H}_{\ell}(\mathrm{u})$ and the definition of $\lambda$.. One finds for: $\nu$ odd $h_{\ell}(\lambda)=0$ while for $\nu$ even

$$
h_{\ell}(\lambda)=\int_{0}^{4 K} H_{\ell}(u) \exp (i \lambda u / 2) d u .
$$

It is this second form of the $h_{\ell}$ integral which will be evaluated. The contour to be used for both integrals is illustrated in Fig. 6. The singularities of $\mathrm{G}_{\ell}(u)$ and $\mathrm{H}_{\ell}(u)$ occur at the same values of $u$ and the relevant ones are indicated by $x$ 's in the figure. Since $G_{\ell}$ and $H_{\ell}$ are finite except at the isolated singularities, the leg of the contour at $\operatorname{Im}(u) \rightarrow \infty$ contributes 
nothing while the vertical legs make no contribution due to the $4 \mathrm{~K}$ periodicity of the integrands. The residues can be evaluated using

$$
\begin{aligned}
& \operatorname{cn} u=\frac{i \pi}{2 k K} \sum_{n=-\infty}^{\infty} \frac{(-1)^{n}}{\sin \left[(\pi / 2 K)\left(u-u_{n}\right)\right]}, d n u=\frac{i \pi}{2 K} \sum_{n=-\infty}^{\infty} \frac{\left.(-1)^{n} \cos [\pi / 2 K)\left(u-u_{n}\right)\right]}{\sin \left[(\pi / 2 K)\left(u \quad u_{n}\right)\right]} \\
& u_{n}=(2 n-1) i K^{\prime} .
\end{aligned}
$$

For the contour of Fig. 6 only the singularities at $u=u_{n}$ with $\mathrm{n} \geq 1$ are relevant. Note that although $\mathrm{cn} u$ and $\mathrm{dn} u$ are singular at $u=2 K+u_{n}$, neither $G_{\ell}(u)$ nor $H_{\ell}(u)$ is singular there when $\ell \geq 0$. Near the singularity $u=u_{n}$, one has

$$
G_{\ell}\left(u+u_{n}\right)=-\frac{2^{\ell+1} i^{\ell}(-1)^{n \ell}}{k\left(u-u_{n}\right)^{\ell+2}}, H_{\ell}\left(u \rightarrow u_{n}\right)=-\left(\frac{2}{k}\right)^{\ell+1} \frac{i^{\ell}(-1)^{n \ell}}{\left(u-u_{n}\right)^{\ell+2}} .
$$

Near the singularities $\mathrm{u}=\mathrm{u}_{\mathrm{n}}, \mathrm{H}_{\ell}(\mathrm{u})=\mathrm{G}_{\ell}(\mathrm{u}) / \mathrm{k}^{\ell}$, consequently for $v$ even

$$
\mathrm{h}_{\ell}(\lambda)=\left(1 / \mathrm{k}^{\ell}\right) \mathrm{g}_{\ell^{(\lambda / 2)}}
$$


and we need evaluate only $g_{\ell}(\lambda)$ : To find the residue $R_{\ell n}$ of $G_{\ell}(u) \exp (i \lambda \dot{u})$ at the singularity $u=u_{n}$, we must expand $\exp (i \lambda u)$ in a Taylor series about $u_{n}$ and keep the $l+1$ term. This gives

$$
\underline{R}_{\ell n}=-i_{j \in} \frac{(2 \lambda)^{\ell+1}}{(L+1) !}(-1)^{(n+1) l} \exp \left(i \lambda u_{n}\right)
$$

In summing the residues $n \geq 1$, one uses the well-known formula

$$
\sum_{n=1}^{\infty} r^{n}=\frac{r}{1-r}
$$

to obtain the required integral

$$
\begin{aligned}
g_{\ell}(\lambda) & =\frac{\pi}{k} \frac{(2 \lambda)^{\ell+1}}{(\ell+1) !} \operatorname{cosech}\left(K^{\prime} \lambda\right), l \text { even } \\
\vdots & \vdots \\
\vdots & \vdots \\
g_{\ell}(\lambda) & =\frac{\pi}{k} \frac{(2 \lambda)^{\ell+1}}{(\ell+1) !} \operatorname{sech}\left(k^{\prime} \lambda\right), \quad l \text { odd } .
\end{aligned}
$$


APPENDIX B

In this appendix the function $H(x)$ will be evaluated for large positive $\mathrm{x}$ with

$$
H(x)=\sum_{\ell=1}^{\infty} \frac{\cdots x^{\ell} \cdots}{(\ell-1) !(\ell:)^{2}}
$$

To obtain the asymptotic form, we use the so-called EulerMaclaurin formula which in our case becomes letting

$$
\begin{aligned}
& f(\ell)=\ln \left(\frac{x^{\ell}}{(\ell-1) !(\ell !)^{2}}\right) \\
& H(x) \equiv \sum_{\ell=1}^{\infty} e^{f(\ell)}=\int_{1}^{\infty} e^{f(\ell)} d \ell+0[f(1)] .
\end{aligned}
$$

For large $x$, the sum is accurately approximated by the integral. The integral can be accurately approximated at large $x$ by the method of steepest descents, which means

$$
H(x) \simeq \int_{-\infty}^{\infty} \exp \left[f_{0}-1 / 2\left|f_{0}^{\prime}\right|\left(\ell-\ell_{0}\right)^{2}\right] d \ell
$$


with $f^{\prime}\left(l_{0}\right)$ equal to zero and $f_{0}$ and $f_{0}^{\prime \prime}$ to function $f(l)$ and its second derivative evaluated at $\ell_{0}$. The factorials in the function $f(\ell)$ are, of course, approximated by sterling's formula. In the limit of large positive $x$ we find

$$
H(x)=\frac{1}{2 \pi \sqrt{3}} \exp \left(3 x^{1 / 3}\right)
$$




\section{REFERENCES}

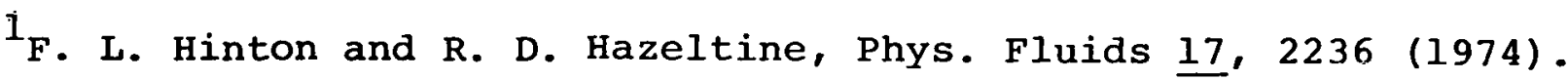

${ }^{2}$ H. Grad, Phys. Fluids 10, 137 (1967).

${ }^{3}$ M. N. Rosenbluth, R. Z. Sagdeev, J. B. Taylor, and G. M. Zaslavskii, Nucl. Fusion 6, 297 (1966).

${ }^{4}$ N. N. Filonenko, R. Z. Sagdeev, and G. M. Zaslavskii, Nucl. Fusion 7,253 (1967).

${ }^{5}$ A. B. Rechester and T. H. Stix, Phys. Rev. Lett. 36,587 (1976).

${ }^{6}$ General reviews on the effect of perturbations on Hamiltonian systems are G. M. Zaslavskii and B. V. Chirikov, Usp. Fiz. Nauk. 10ㄷ, 3 (1971) (Sov. Phys. Uspekhi 14, 549 (1972), and Radu Balescu, Equilibrium and Non-Equilibrium Statistical Mechanics (Wiley, New York, 1975) p. 695.

7 J. Hosea, private communication. 


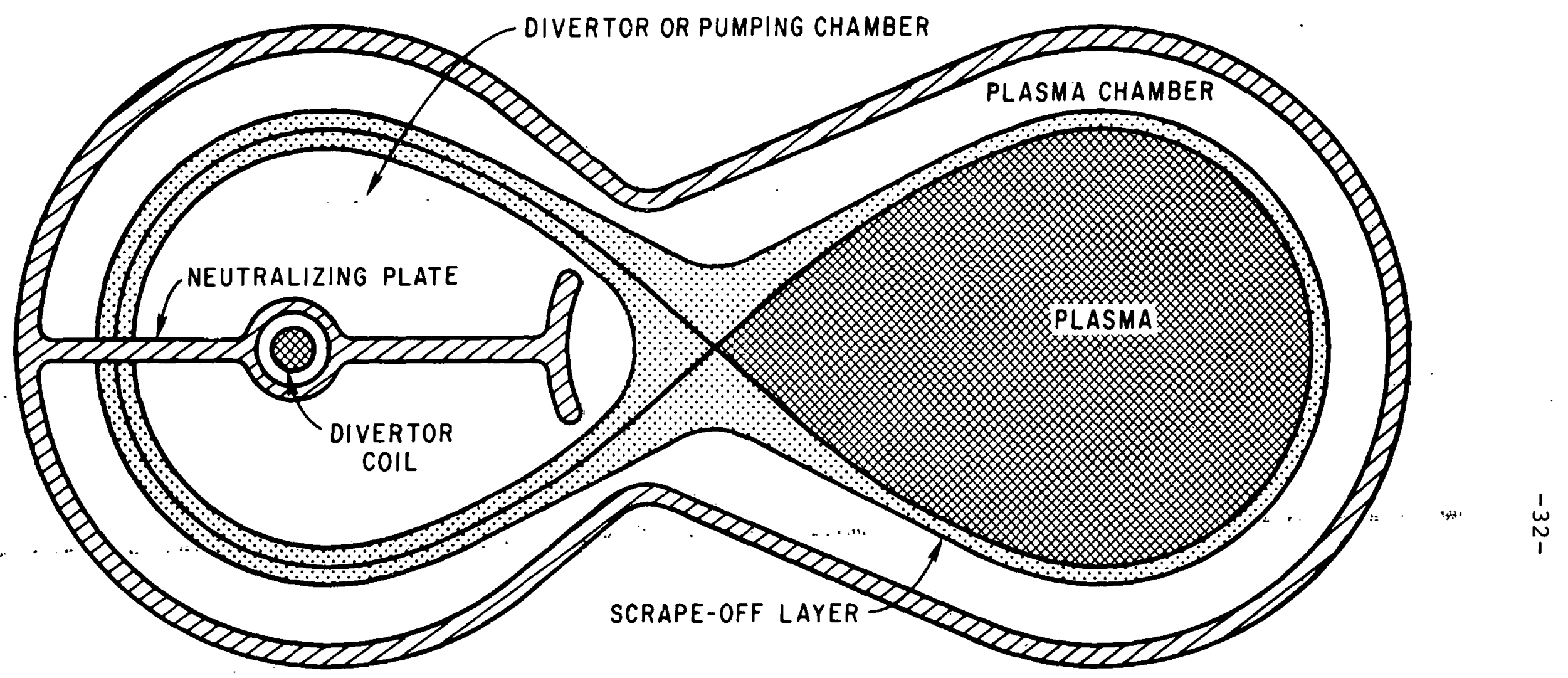

772149

Fig. 1. A tokamak with a divertor. 


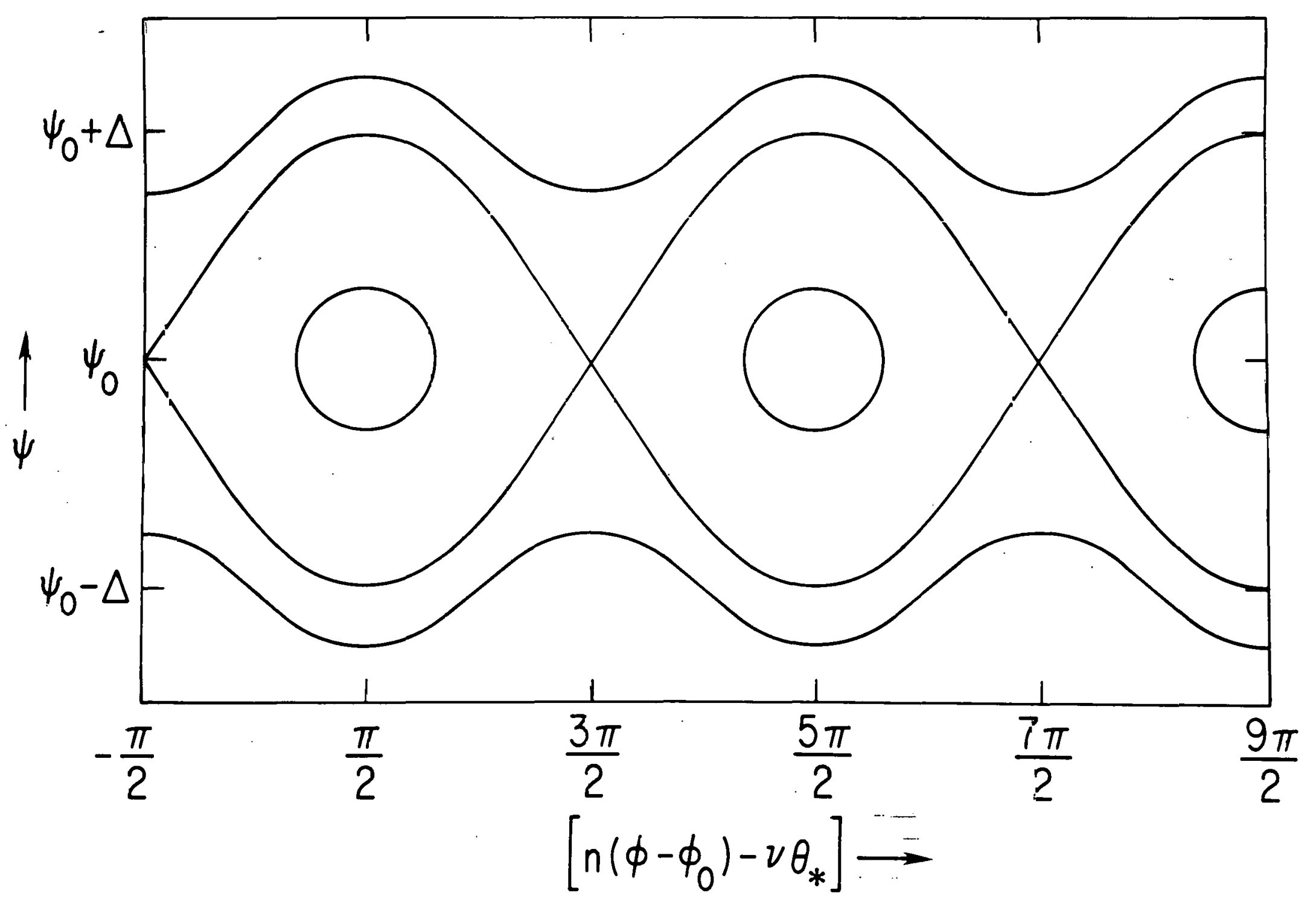

72085

Fig. 2. A magnetic island chain. The island chain is $q\left(\psi_{0}\right)=\nu / n$ with $\nu$ and $n$ intergers If the surfaces were customary notation, be $m \theta$. 


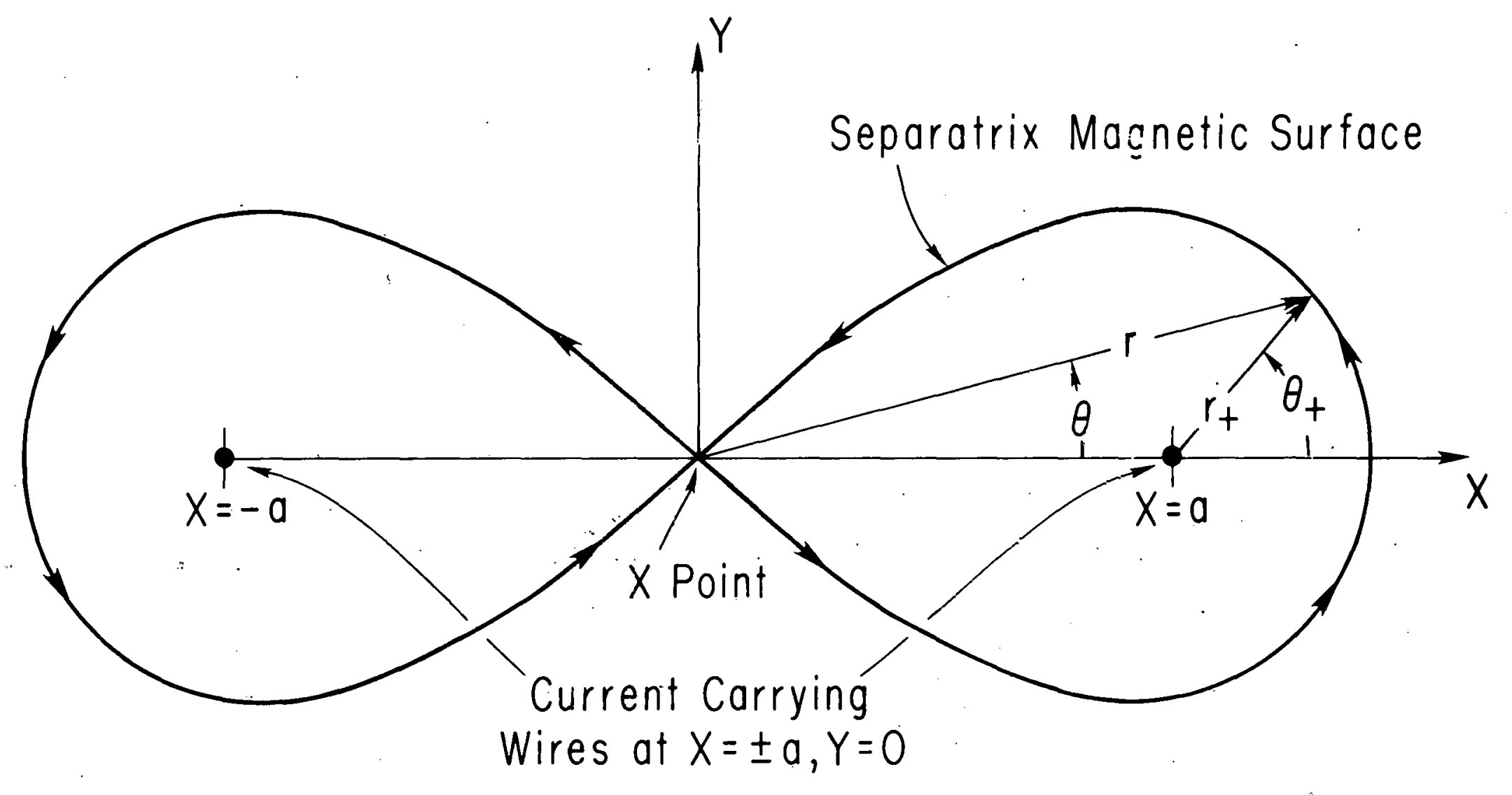

Fig. 3. Two-wire divertor model. This 772083 currents flowing in wires model has equal cylindrical coordinate sytems are at $x= \pm a, y=0$. Two an $r, \theta$ system about the system about the wire at $\mathrm{x}=\mathrm{a}, \mathrm{y}=0 . \mathrm{y}=0)$ and a. $\mathrm{r}_{+}, \theta_{+}$ 


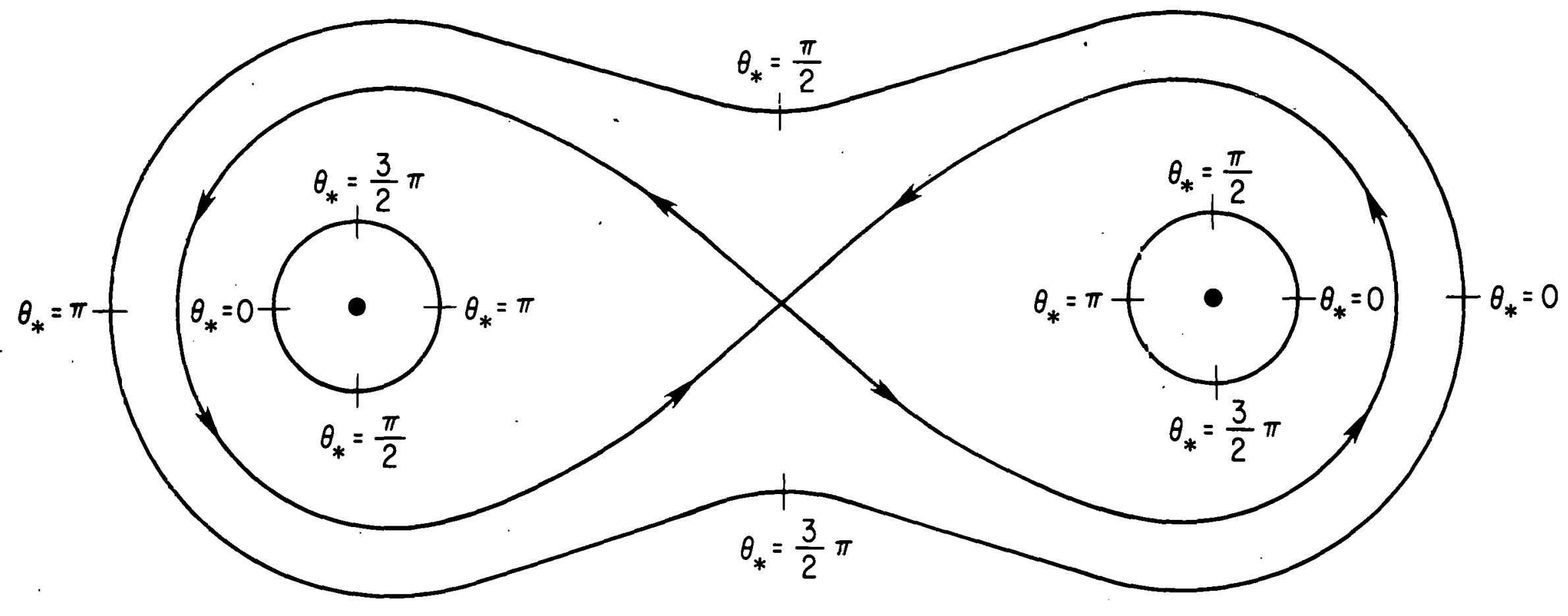

Fig. 4. The quadrant conventions for $\theta_{\star}$. 


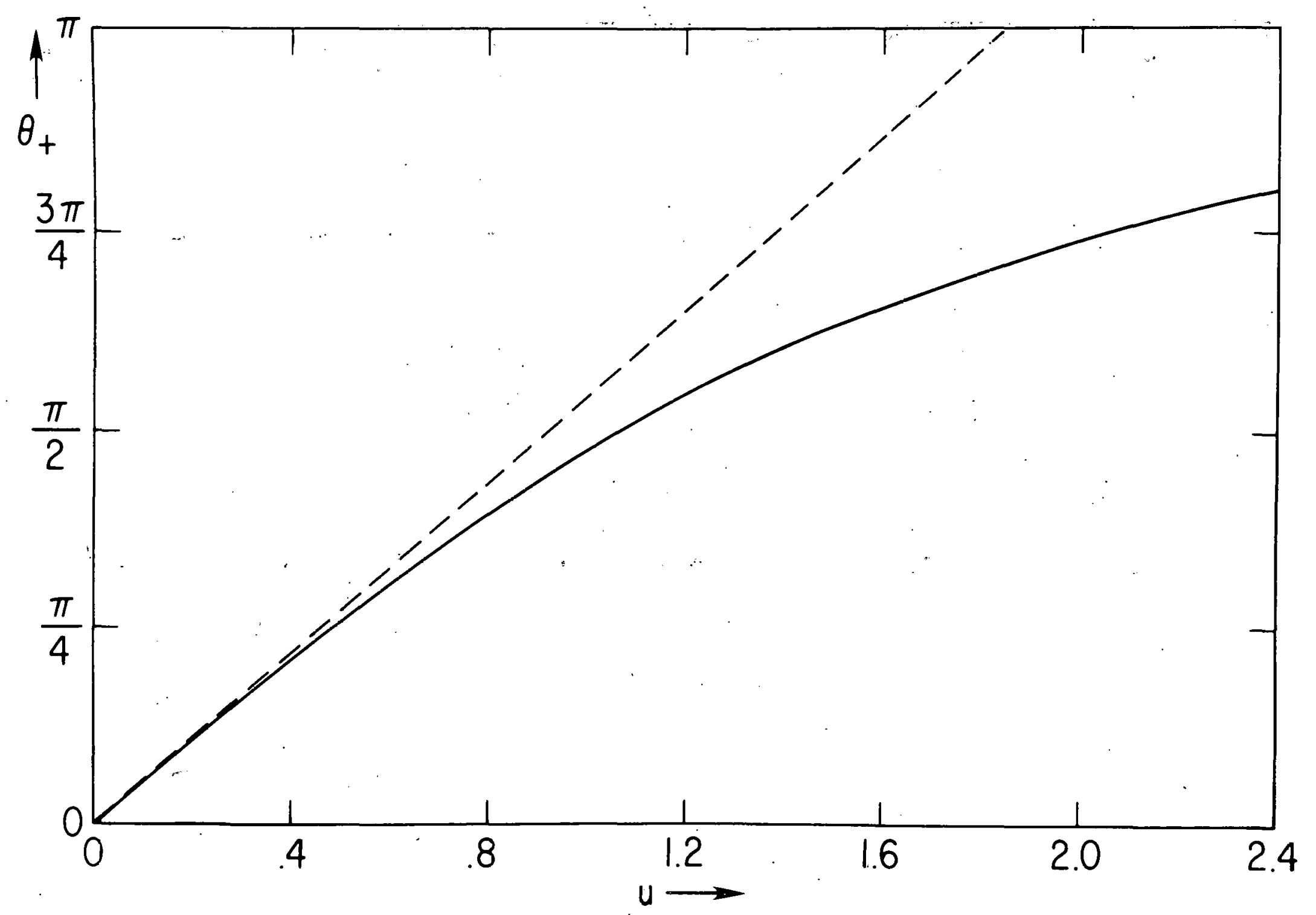
the approximate linear form used ${ }^{+}$in the paper. 


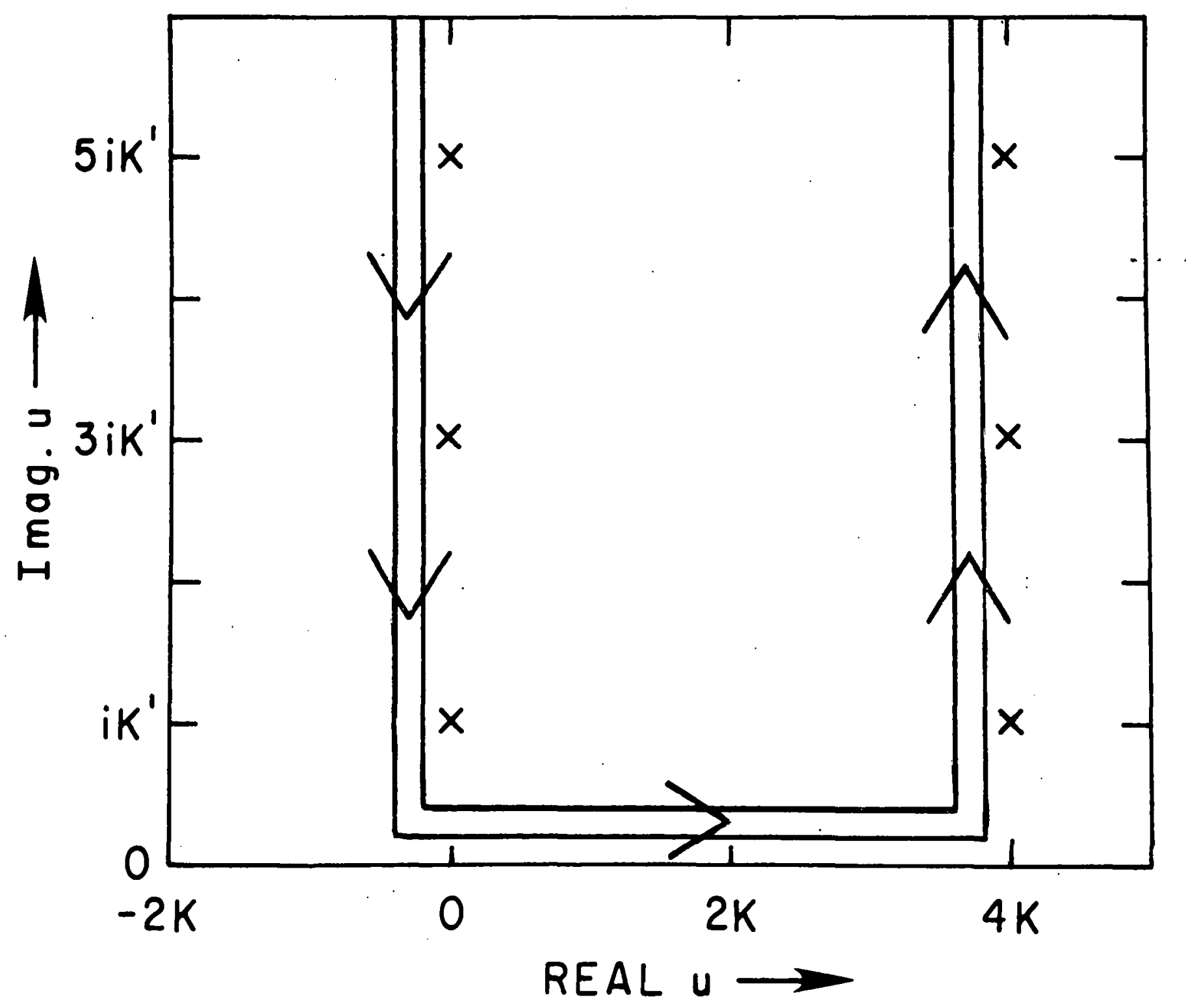

772081

Fig. 6. The contour for the integrals $g_{l}$ and $h_{2}$. The $x$ 's on the diagram indicate the singularities of the integrand. 